Doug Geisler, Eva K. Grebel, and Dante Minniti, eds.

\title{
Kinematic Masses of Super Star Clusters in NGC 1569
}

\author{
Andrea M. Gilbert \& James R. Graham \\ University of California, 601 Campbell Hall, Berkeley, CA 94720-3411
}

\begin{abstract}
We summarize recent results from our study of the massive young super star clusters (SSCs) in the dwarf galaxy NGC 1569, and present new high-resolution NIRSPEC spectra that permit the measurement of a cluster's intrinsic stellar velocity dispersion. Thus we derive kinematic masses for the two brightest SSCs: $2.3 \times 10^{5} \mathrm{M}_{\text {sun }}$ for SSC B, and $3.9 \times 10^{5}$ and $4.4 \times 10^{5} \mathrm{M}_{\text {sun }}$ for the two components of SSC A.
\end{abstract}

\section{Introduction}

Resolved starbursts in a variety of environments appear to form stars preferentially in a clustered mode, generating only a few, or up to a few 1000 massive young super star clusters (SSCs). High angular resolution HST observations have shown that SSCs have radii comparable with those of globular clusters (e.g. Whitmore et al. 1999), and a few spectroscopic observations of cluster velocity dispersions have shown that at least some SSCs are bound, with kinematic masses of $10^{5}-10^{6} \mathrm{M}_{\text {sun }}$ comparable to those of globulars (e.g. Ho \& Filippenko 1996). Their brightnesses $\left(<\mathrm{M}_{\mathrm{V}}>\approx-11 \mathrm{mag}\right.$ in the Antennae, Whitmore \& Schweizer 1995) are consistent with those expected for globulars at the young ages of a few to a few 10s of Myr inferred for SSCs. The evidence suggests that these objects are protoglobular clusters forming in the present-day universe.

The dwarf irregular galaxy NGC 1569 is one of the nearest (at $2.2 \mathrm{Mpc}$, ref) recent starbursts that harbors some of these objects, which are inferred from colors and spectroscopy to have ages of 5-20 Myr (e.g. Origlia et al. 2001, and references therein). It shows many additional signs of recent star formation, including Wolf-Rayet emission, HII regions, a halo of disturbed diffuse ionized gas in shells and filaments, and an x-ray wind, along with both atomic and molecular gas still present.

We present the latest results from our program of infrared spectroscopy at low and high spectral resolution of the brightest SSCs in nearby starbursts. In previous contributions we have discussed techniques for analyzing cluster stellar populations using such spectra together with model atmospheres, empirical spectral libraries, and population synthesis models (Gilbert \& Graham 2000; 2001a), and applied them to the SSCs of NGC 1569. We have shown that broad wavelength coverage in high signal-to-noise ratio near-IR spectra can be used to identify the dominant spectral type of a cluster. Fitting the photospheric spectrum of a SSC to a grid of model stellar atmospheres (from Hauschildt, Allard, \& Baron 1999) characterizes the effective $\mathrm{T}_{\text {eff }}$ and $\log [\mathrm{g}]$ of the cluster. We calculated infrared synthetic cluster spectra from empirical stellar spectra 


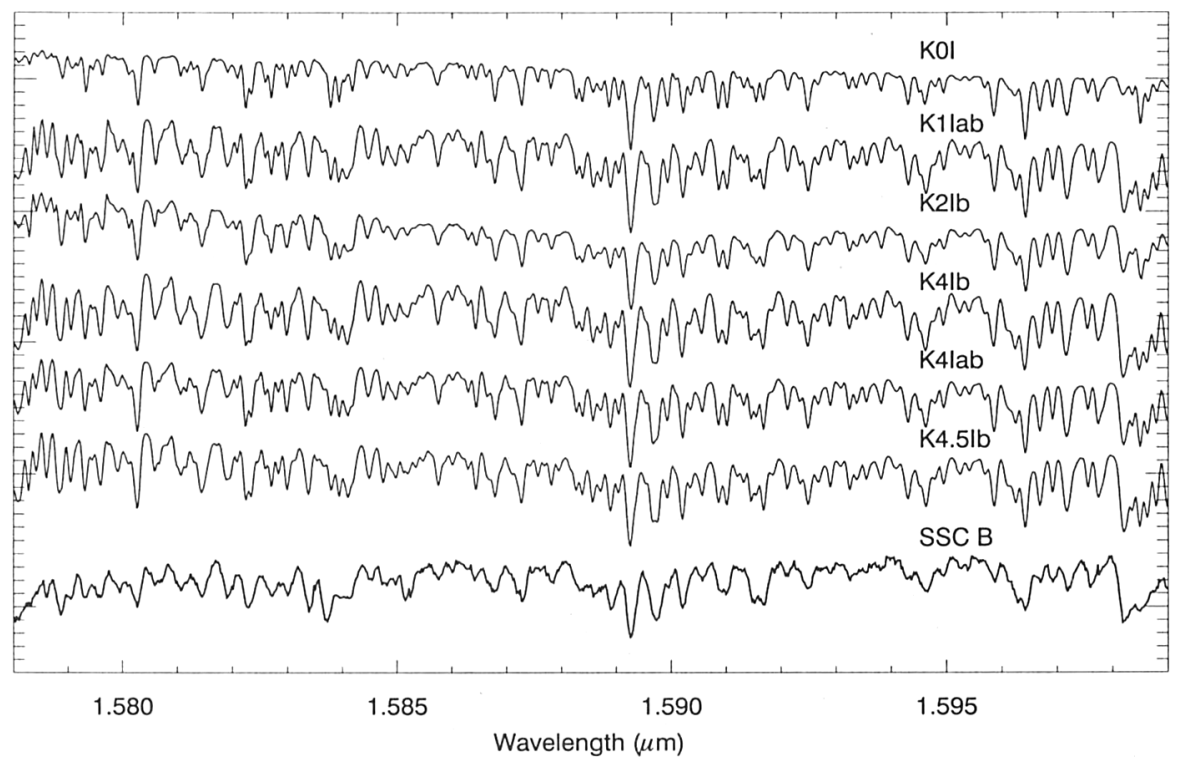

Figure 1. Sequence of supergiant spectra in NIRSPEC order 48 (labelled with optical spectral classifications) compared with spectrum of SSC B at bottom. Note the width of features in the cluster and the prominent CO $5-2$ bandhead near $1.598 \mu \mathrm{m}$.

(Wallace \& Hinkle 1996; Meyer et al. 1998) and Starburst99 population synthesis models (Leitherer et al. 1999), which help constrain the age of a cluster. Here we briefly summarize new work on measuring the intrinsic velocity dispersions of the NGS 1569 SSCs from high-resolution NIRSPEC spectra. A more complete discussion will appear in Gilbert \& Graham (2001b) and Graham \& Gilbert (2001).

\section{Measuring the Kinematic Masses of SSCs}

We obtained NIRSPEC echelle observations (at $\mathrm{R} \approx 24,000$ ) of the NGC 1569 SSCs and of late-type giant and supergiant stars in Dec. 2000, Feb. 2001, and Jun. 2001. A sequence of K-supergiant spectra (one of the 7 orders in the $H$-band echellogram), ordered by optical spectral type, is shown in Fig. 1 along with the spectrum of SSC B. The high signal-to-noise ratio stellar spectra strongly resemble the cluster spectrum, though the latter shows clear velocity broadening of the features due to the intrinsic velocities of the cluster stars.

We measured the line-of-sight velocity dispersion $\sigma$ of SSCs A and B using cross-correlation between the cluster and stellar spectra. Fig. 2 displays the cross-correlation functions (XCFs) between the cluster and a K5-M0 supergiant together with the autocorrelation function (ACF) of the star. SSC B has a symmetric, resolved XCF that gives $\sigma=7.5 \pm 0.2 \mathrm{~km} / \mathrm{s}$, which implies a kinematic 

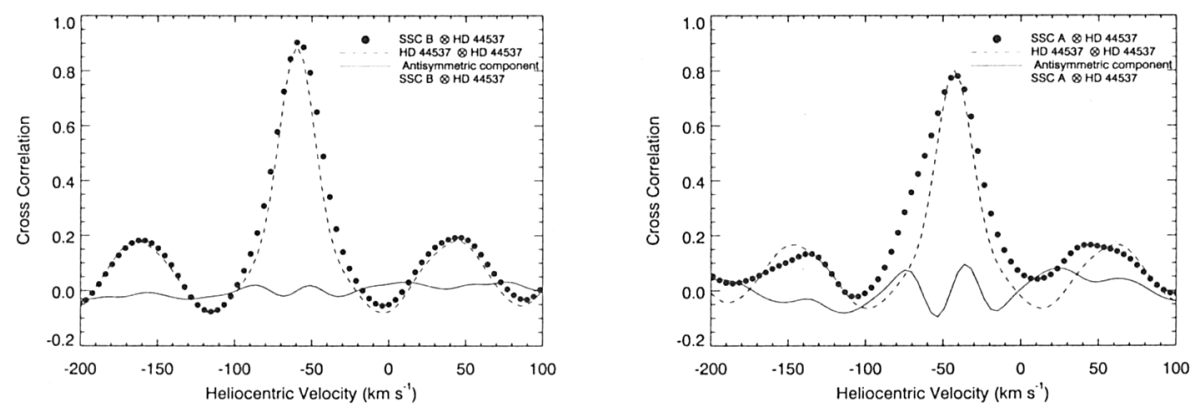

Figure 2. Left: XCF (dots) for SSC B with a K5-M0 supergiant star is resolved by the stellar autocorrelation profile (dashes). Measured 1D cluster velocity dispersion is $7.5 \mathrm{~km} / \mathrm{s}$. Right: As above but for SSC $\mathrm{A}$, whose $\mathrm{XCF}$ is antisymmetric and is best fit by two kinematic components with $10.4 \mathrm{~km} / \mathrm{s}$ velocity dispersions separated by $21.4 \mathrm{~km} / \mathrm{s}$.

mass of $2.3 \times 10^{5} \mathrm{M}_{\text {sun }}$, using the virial relation $M=9.8 \sigma^{2} r_{h P} / G$, where $r_{h P}$ is the projected half-light radius (Spitzer 1987). SSC A has an antisymmetric XCF that is best fit by two gaussian components separated by $21.4 \mathrm{~km} / \mathrm{s}$, each with $\sigma=10.4 \pm 0.4 \mathrm{~km} / \mathrm{s}$. This implies kinematic masses for the two components of $3.9 \times 10^{5}$ and $4.4 \times 10^{5} \mathrm{M}_{\text {sun }}$. This may be compared with a total mass for SSC A of about $10^{6} \mathrm{M}_{\text {sun }}$ derived from the $\sigma$ measured by Ho \& Filippenko (1996), where they assumed a single kinematic component.

Acknowledgments. AMG acknowledges support from a NASA GSRP Fellowship.

\section{References}

Gilbert, A. M. \& Graham, J. R. 2000, to appear in proceedings of Ringberg Workshop: Starbursts - Near \& Far (Springer)

Gilbert, A. M. \& Graham, J. R. 2001a, BAAS \#198

Gilbert, A. M. \& Graham, J. R. 2001b, in preparation

Graham, J. R. \& Gilbert, A. M. 2001, in preparation

Hauschildt, P. H., Allard, F., \& Baron, E. 1999, ApJ, 512, 377

Ho, L. C., \& Filippenko, A. V. 1996, ApJ, 466, L83

Leitherer, C. et al. 1999, ApJS, 123, 3

Meyer, M. R., Edwards, S., Hinkle, K. H., \& Strom, S. E. 1998, ApJ, 508, 397

Origlia, L., et al. 2001, astro-ph/0105195

Spitzer, L. 1987, Dynamical Evolution of Globular Clusters (Princeton: Prinction Univ. Press), 13

Wallace, L., \& Hinkle, K. H. 1996, ApJS, 107, 312

Whitmore, B. C., \& Schweizer, F. 1995, AJ, 109, 960

Whitmore, B. C., et al. 1999, AJ, 118, 1551 